\title{
Esophageal Leiomyomatosis Diagnosed by Endoscopic Ultrasound
}

\section{R. Sotoudehmanesh ${ }^{1}$, A. Ghafoori ${ }^{2}$,}

\section{J. Mikaeli', S. M. Tavangar ${ }^{3}$,}

\section{H. Mohajeri Moghaddam²}

${ }^{1}$ Digestive Diseases Research Center, Tehran University of Medical Sciences, Tehran, Iran

${ }^{2}$ Department of Surgery, Shariati Hospital, Tehran University of Medical Sciences, Tehran, Iran

${ }^{3}$ Department of Pathology, Shariati Hospital, Tehran University of Medical Sciences, Tehran, Iran

\section{Corresponding Author}

\section{R. Sotoudehmanesh, M.D.}

Digestive Diseases Research Center,

Shariati Hospital

North Kargar Avenue

14114 Tehran

Iran

Fax: $\quad+98-21-8012992$

E-mail: setoodeh@ams.ac.ir

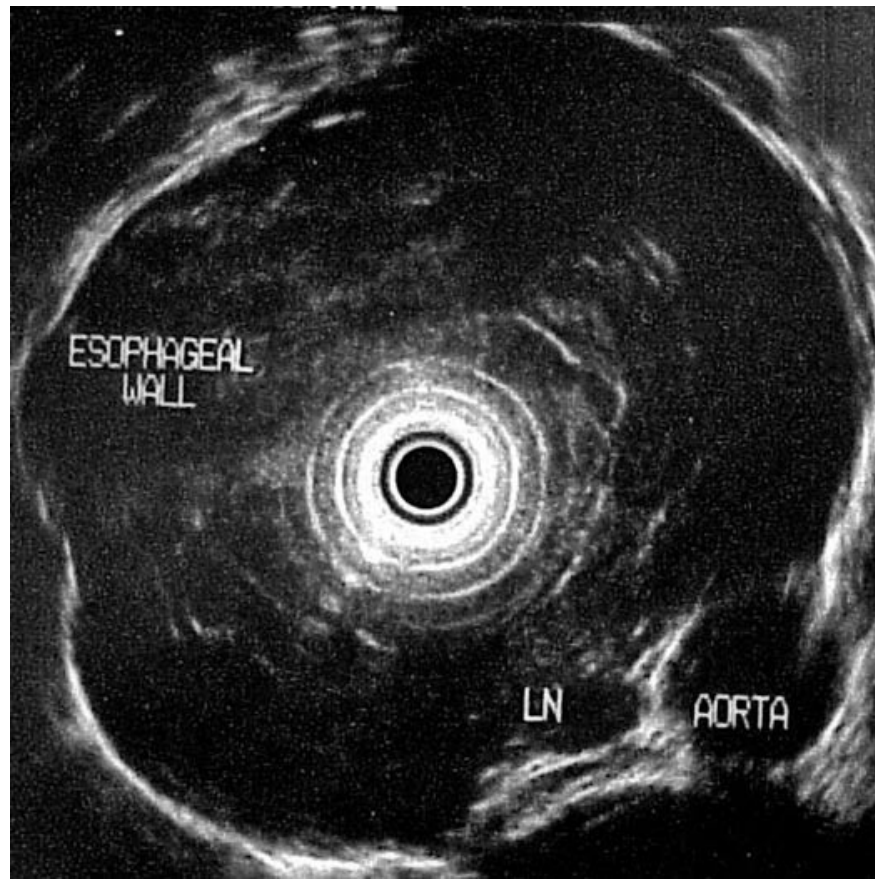

Figure 1 Endoscopic ultrasound showed multifocal wall thickness of varying size $(1-3 \mathrm{~cm})$ originating from the muscularis propria layer, in a 39-year-old lady presenting with dysphagia and regurgitation that had been slowly progressive for 10 years. Barium swallow showed irregularity in the lower two-thirds of esophagus. Nodularity of the esophagus with tortuosity was found at endoscopy. Compare the size of the esophagus with the adjacent aorta.

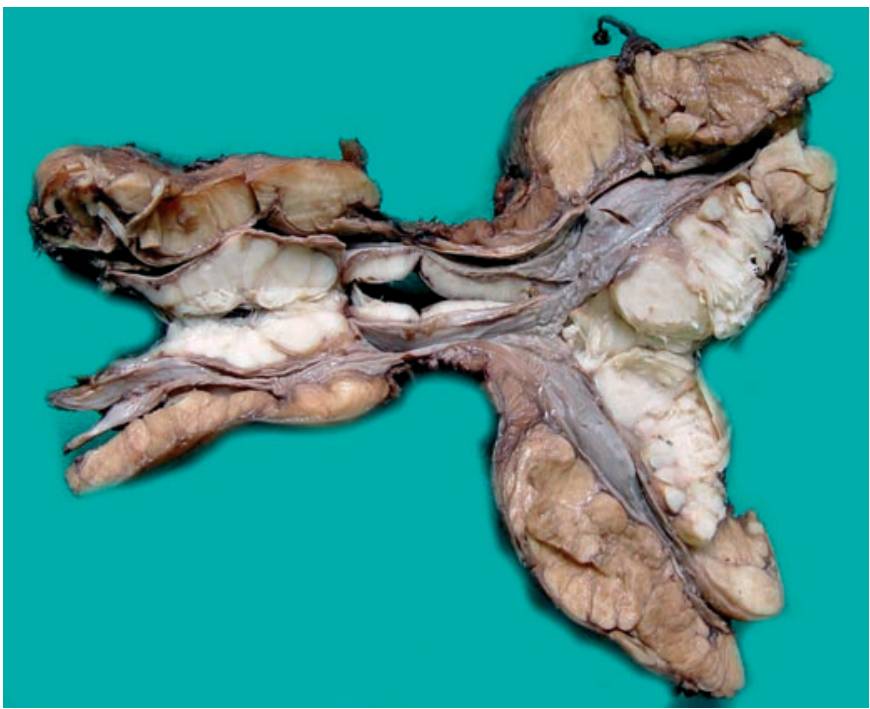

Figure 2 Esophagectomy was done and histopathological investigation revealed esophageal leiomyomatosis. The resected esophagus had multiple stromal tumors of varying sizes. The patient is now symptom-free. 